International Journal of Engineering \& Technology, $7(1.3)(2018) 56-60$
International Journal of Engineering \& Technology
WPC
Website: www.sciencepubco.com/index.php/IJET
Review paper

\title{
Designing modular and distributive lattices using $\ell$-soft group: A survey
}

\author{
J.Vimala ${ }^{1^{*}}$ and L.Vijayalakshmi ${ }^{2}$ \\ ${ }^{1}$ Assistant Prof., Department of Mathematics, Alagappa University, Karaikudi India \\ ${ }^{2}$ Research Scholar, Department of Mathematics, Alagappa University, Karaikudi India \\ *vimaljey@alagappauniversity.ac.in
}

\begin{abstract}
Soft group was firstly introduced by Aktas and Cagmam. The concept of modular and distributive soft lattices over the soft sets was offered by Faruk Karaaslan. In this paper, we discuss the modular and distributive lattices on soft group. We study several characterizations of modular and distributive lattice ordered soft group ( $\ell$ - soft group) and derived its properties.
\end{abstract}

Keywords: Soft sets, Soft groups, Lattice ordered soft sets, Modular $\ell$ - soft group, Distributive $\ell$ - soft group

\section{Introduction}

A brief foundation to the distributive lattice theory with pseudocomplementation has been conferred by Gratzer [10]. Molodtsov[13] suggested a new concept of soft set theory, to overcome the difficulties that existing theories with uncertainty. Soft set theory has been emerged as a mathematical tool to solve complicated problems with uncertainties. Molodtsov described many directions for the application of soft sets. Then Maji et al.[12] proposed several operations and presented the application of soft set theory. The fundamentals of soft set theory was summarized by I.A.Onyeozili and T.M. Gwary[16]. Grzegorz Dymek and Andrzej Walendziak [11] initiated the notion of soft general algebra, soft subalgebra and also studied its properties. The concept of distributive and modular soft lattices was discussed by Faruk Karaaslan et.al. [8]. The notion of soft group was conferred by Aktas and Cagman [1]. Xia Yin and Zuhua Liao[21] studied about the concept of soft groups. The lattice structure of the soft group was offered by Yingehao Shao and Keyun Qin[22]. M.I.Ali et al.[4] introduced the lattice ordered soft set and obtained some of its properties. R.Natarajan and J.Vimala[15] propounded distributive l-ideal in a commutative l-group. J.Arockia Reeta and J.Vimala[3, 19] investigated the distributive and modular properties on lattice ordered fuzzy soft group and also discussed its duality. The concept of lattice ordered soft group ( $\ell$ - soft group) was initiated and its properties were studied by L.Vijayalakshmi and J.Vimala[17]. E.K.R.Nagarajan and P.Geetha[14] defined the modular and distributive soft lattice and established its characterization theorems. Characterizations of modular and distributive semilattices were discussed by W.H. Cornish[7]. Fuzzy $l$-group and Lattices of Fuzzy $l$-ideal were studied by J.Vimala et.al., $[5,6,18]$. Fuzzy soft cardinality in lattice ordered fuzzy soft group has been successfully applied in decision making problems by J.Vimala et.al.[20]

In this paper, we initiate the notions of modular $\ell$ - soft group and distributive $\ell$ - soft group and also establish some results on modular $\ell$ - soft group and distributive $\ell$ - soft group with respect to soft homomorphism and soft group $\ell$-ideal on $\ell$ - soft group.

This paper is arranged as follows: In sec 2 , shortly reviews some basic definitions of soft set, soft group, lattice ordered soft set and $\ell$ - soft group. In sec 3 , we study the properties of modular $\ell$ - soft group and distributive $\ell$ - soft group on $\ell$ - soft group.

\section{Preliminaries}

In this section, we recall some basic definitions of lattices, soft sets and soft groups.

Let us first define a lattice in an algebraic way.

[9] An algebraic lattice $(L, \wedge, \vee)$ is a set $L$ with two binary operations meet and join, $\wedge$ and $\vee$ such that both operations are commutative and associative, and the absorption law holds. that is $\forall a, b, c \in L$,

1. $a \wedge b=b \wedge a, a \vee b=b \vee a$ (Commutivity)

2. $(a \wedge b) \wedge c=a \wedge(b \wedge c),(a \vee b) \vee c=a \vee(b \vee c)$ (Associativity)

3. $a=a \wedge(a \vee b)=a \vee(a \wedge b)$ (Absorption law)

In an algebraic lattice, both operations are idempotent.

That is, $\forall a \in L, a \wedge a=a \vee a=a$.

In an algebraic lattice $\mathrm{L}$, for any $a, b \in L$, we have $a \vee b=a$ iff $a \wedge b=b$.

A sublattice is a nonempty subset L' of an algebraic lattice L, such that $\mathrm{L}^{\prime}$ is closed under joint and meet.

A partial order is a relation ' $\geq^{\prime}$ on a set $\mathrm{S}$ that is reflexive, antisymmetric and transitive. That is $\forall a, b, c \in S$,

1. $a \geq a$

2. $a \geq b, b \geq a$ implies $a=b$

3. $a \geq b, b \geq c$ implies $a \geq c$

An order lattice is a poset $(L, \geq)$ such that for any $a, b \in L, \sup \{a, b\}$ and $\inf \{a, b\}$ exist.

Definition 2.1. [13] Let $U$ be an initial universe set and $E$ a set of parameters with respect to $U$. Let $P(U)$ denote the power set of $U$ 
and $A \subseteq E$. For the soft group, universe be a group. A pair $(F, A)$ is called a soft set over $U$, where $F$ is a mapping given by $F: A \rightarrow P(U)$. In other words, a soft set $(F, A)$ over $U$ is a parameterized family of subsets of $U$. For $e \in A, F(e)$ may be considered as the set of e-elements or e-approximate elements of the soft set $(F, A)$.

Definition 2.2. [13] Let $(F, A)$ and $(H, B)$ be two soft sets over common universe $U$, we say that $(H, B)$ is said to be a soft subset of $(F, A)$ if

1. $A \subseteq B$ and

2. $F(e) \subseteq H(e)$ for all $e \in A$.

We write $(H, B) \tilde{C}(F, A)$, and $(H, B)$ is said to be a soft super set of $(F, A)$, if $(F, A)$ is a soft subset of $(H, B)$. We denote it by $(H, B) \tilde{\supset}(F, A)$

Definition 2.3. [13] Two soft sets $(F, A)$ and $(H, B)$ over a common universe $U$ are said to be soft equal if $(F, A)$ is a soft subset of $(H, B)$ and $(H, B)$ is a soft subset of $(F, A)$.

Definition 2.4. [12] Let $(F, A)$ and $(G, B)$ be two soft sets over a common universe $U$. Then the union of $(F, A)$ and $(G, B)$ denoted $(F, A) \cup(G, B)$ is a soft set $(H, C)$, where $C=A \cup B$ and for all $e \in C$, $H(e)= \begin{cases}F(e), & \text { if } e \in A-B \\ G(e), & \text { if } e \in B-A \\ F(e) \cup G(e), & \text { if } e \in A \cap B\end{cases}$

Definition 2.5. [12] Let $(F, A)$ and $(G, B)$ be two soft sets over a common universe $U$. Then the intersection of $(F, A)$ and $(G, B)$ denoted $(F, A) \tilde{\cap}(G, B)$ is a soft set $(H, C)$, where $C=A \cap B$ and $\forall e \in C, H(e)=F(e)$ or $G(e)$ (as both are same set).

Definition 2.6. [1] Let $G$ be a group and $E$ be a set of parameters. For $A \subseteq E$, the pair $(F, A)$ is called a soft group over $G$ if and only if $F(a)$ is a subgroup of $G$ for all $a \in A$, where $F$ is a mapping of $A$ into the set of all subsets of $G$.

Definition 2.7. [4] If $U$ is an initial universe, then $P(U)$ is a bounded lattice and the set of parameters $E$, is also a lattice with respect to certain binary operations (or) partial order and $A \subseteq E$. A soft set $(F, A)$ is called a lattice ordered soft set if for the mapping $F: A \rightarrow P(U), x \leq y$ implies $F(x) \subseteq F(y)$, for all $x, y \in A \subseteq E$.

Definition 2.8. [17] Let $G$ be a group and $P(G)$ be the power set of $G$. Let $E$ be the set of parameters(lattice), $A \subseteq E$. Then a soft set $(F, A)$ is said to be a lattice ordered soft group $(\ell-$ soft group) iffor the mapping $F: A \rightarrow P(G)$,

1. $F(a)$ is a subgroup of $G$, for all $a \in A$.

2. For all $a, b \in A, a \vee b, a \wedge b \in A$ then $\operatorname{Sup}\{F(a), F(b)\}$ and Inf $\{F(a), F(b)\}$ exist in $(F, A)$.

Note:Throughout this section, $F(a) \vee F(b)$ and $F(a) \wedge F(b)$ are used for $\operatorname{Sup}\{F(a), F(b)\}$ and $\operatorname{Inf}\{F(a), F(b)\}$ respectively. Also $\vee$ denotes $\cup, \wedge$ denotes $\cap$ and $\mathrm{L}$ denotes the set of all $\ell$ - soft groups.

\section{Modular $\ell$ - soft group and Distributive $\ell$ - soft group}

Definition 3.1. (i) For any three elements of soft set $(F, A)$, the modular law is $[F(a) \wedge F(b)] \vee[F(a) \wedge F(c)]=F(a) \wedge[(F(a) \wedge F(b)) \vee$ $F(c)]=F(a) \wedge[F(b) \vee(F(a) \wedge F(c))]$, if $F(a) \subseteq F(b)$ and $[F(a) \vee F(b)] \wedge[F(a) \vee F(c)]=F(a) \vee[(F(a) \vee F(b)) \wedge F(c)]=$ $F(a) \vee[F(b) \wedge(F(a) \vee F(c))]$, if $F(a) \supseteq F(b)$.

An $\ell$ - soft group in which the modular law holds is a modular $\ell$ soft group.

(ii) The distributive law is $[F(a) \wedge F(b)] \vee[F(a) \wedge F(c)]=F(a) \wedge$ $[F(b) \vee F(c)]$ and

$[F(a) \vee F(b)] \wedge[F(a) \vee F(c)]=F(a) \vee[F(b) \wedge F(c)]$.

An $\ell$ - soft group in which the distributive law holds is a distributive $\ell$ - soft group.
Note:

(i) If $(F, A)$ is modular $\ell$ - soft group, then $F(a) \wedge[F(b) \vee F(c)]=$ $F(b) \vee[F(a) \wedge F(c)]$,

$\forall F(a), F(b), F(c) \in(F, A)$.

(ii) If $F(a)=F(b)$, then $F(a) \wedge[F(b) \vee F(c)]=F(a) \wedge[F(a) \vee$ $F(c)]=F(a)$ and $F(b) \wedge[F(a) \vee F(c)]=F(a) \wedge[F(a) \vee F(c)]=$ $F(a)$.

(iii) Dual modular $\ell$ - soft group $F(a) \vee[F(b) \wedge F(c)]=$ $F(b) \wedge[F(a) \wedge F(c)]$

(iv) If $(F, A)$ is distributive $\ell$ - soft group, then $F(a) \wedge[F(b) \vee F(c)]=$ $[(F(a) \wedge F(b)] \vee F(a) \wedge F(c)], \forall F(a), F(b), F(c) \in(F, A)$.

Example 3.2. (i)Let $(F, N)=\left\{3^{n} z: n \in N, z \in Z\right\}$ be an $\ell$ - soft group.

Take $(F, N)=\left\{3^{2} z, 3^{3} z, 3^{4} z\right\}$, if $F(a) \supseteq F(b)$.

$F(a) \wedge[F(b) \vee F(c)]=\{9 z\} \cap[\{27 z\} \cup\{81\}]$

$=\{9 z\} \cap\{27 z\}$

$=\{27 z\}$

$F(b) \vee[F(a) \wedge F(c)]=\{9 z\} \cup[\{27 z\} \cap\{81\}]$

$=\{9 z\} \cup\{27 z\}$

$=\{27 z\}$

Hence $(F, N)$ is a modular $\ell$ - soft group.

(ii) $(F, N)=\left\{z_{n}: n \in N, z \in Z\right\}$, if $F(a) \subseteq F(b)$ is a modular $\ell$ - soft group.

Proposition 3.3. $(F, A)$ is distributive $\ell$ - soft group iff $(F, A)$ is modular $\ell$ - soft group

Proof.

Let $(F, A)$ is distributive $\ell$ - soft group and $\forall F\left(a_{1}\right), F\left(a_{2}\right), F\left(a_{3}\right) \in$ $(F, A)$.

Now $\left[F\left(a_{1}\right) \vee F\left(a_{2}\right)\right] \wedge\left[F\left(a_{1}\right) \vee F\left(a_{3}\right)\right]$

$=\left\{\left[F\left(a_{1}\right) \vee F\left(a_{2}\right)\right] \wedge F\left(a_{1}\right)\right\} \vee\left\{\left[F\left(a_{1}\right) \vee F\left(a_{2}\right)\right] \wedge F\left(a_{3}\right)\right\}$

$=F\left(a_{1}\right) \vee\left\{\left[F\left(a_{1}\right) \vee F\left(a_{2}\right)\right] \wedge F\left(a_{3}\right)\right\}$ (by absorption)

$=F\left(a_{1}\right) \vee\left\{\left[F\left(a_{1}\right) \wedge F\left(a_{3}\right)\right] \vee\left[F\left(a_{2}\right) \wedge F\left(a_{3}\right)\right]\right\}$

$=\left\{F\left(a_{1}\right) \vee\left[F\left(a_{1}\right) \wedge F\left(a_{3}\right)\right]\right\} \vee\left[F\left(a_{2}\right) \wedge F\left(a_{3}\right)\right]$

$=F\left(a_{1}\right) \vee\left[F\left(a_{2}\right) \wedge F\left(a_{3}\right)\right]$

Conversely, let $(F, A)$ is modular $\ell$ - soft group, then

$\left[F\left(a_{1}\right) \wedge\left[F\left(a_{2}\right) \vee F\left(a_{3}\right)\right]=F\left(a_{1}\right) \wedge\left[F\left(a_{3}\right) \vee F\left(a_{2}\right)\right]\right.$

$=\left\{F\left(a_{1}\right) \wedge\left[F\left(a_{3}\right) \vee F\left(a_{1}\right)\right]\right\} \wedge\left[F\left(a_{3}\right) \vee F\left(a_{2}\right)\right]$ (by absorption)

$=F\left(a_{1}\right) \wedge\left\{\left[F\left(a_{3}\right) \vee F\left(a_{1}\right)\right] \wedge\left[F\left(a_{3}\right) \vee F\left(a_{2}\right)\right]\right\}$

$=F\left(a_{1}\right) \wedge\left\{\left[F\left(a_{1}\right) \wedge F\left(a_{2}\right)\right] \vee F\left(a_{3}\right)\right\}$

$=\left[F\left(a_{1}\right) \wedge F\left(a_{2}\right)\right] \vee\left[F\left(a_{1}\right) \wedge F\left(a_{3}\right)\right]$

Therefore $(F, A)$ is distributive $\ell$ - soft group.

Definition 3.4. If $(F, A)$ is a poset in which every two subgroups of $(F, A)$ are comparable it is called a soft group toset.

Example 3.5. A soft group toset is a modular $\ell$ - soft group.

Proposition 3.6. A sub $\ell$ - soft group of a modular $\ell$ - soft group is modular $\ell$ - soft group.

Proof.

Let $(F, A)$ be a modular $\ell$ - soft group and $(G, B) \subseteq(F, A)$.

If $G\left(b_{1}\right), G\left(b_{2}\right), G\left(b_{3}\right) \in(G, B)$ with $G\left(b_{1}\right) \supseteq G\left(b_{2}\right) \supseteq G\left(b_{3}\right)$, then $G\left(b_{1}\right) \wedge\left[G\left(b_{2}\right) \vee G\left(b_{3}\right)\right]=G\left(b_{2}\right) \vee\left[G\left(b_{1}\right) \wedge G\left(b_{3}\right)\right]$.

since $(G, B)$ is a $\ell$-soft group under $\vee$ and $\wedge$, this result holds in $(G, B)$.

Hence $(G, B)$ is modular $\ell$ - soft group.

Definition 3.7. Let $(F, A)$ and $(G, B)$ be two $\ell$-soft groups. Let $\Psi:(F, A) \rightarrow(G, B)$ and $\Theta: A \rightarrow B$. Then $(\Psi, \Theta)$ is said to be $\ell$ - soft group homomorphism, if indent $(i) \Theta$ is onto

(ii) $\Psi\left[F\left(e_{i}\right) \vee F\left(e_{j}\right)\right]=\Psi\left[F\left(e_{i}\right)\right] \vee \Psi\left[F\left(e_{j}\right)\right]$, and $\Psi\left[F\left(e_{i}\right) \wedge F\left(e_{j}\right)\right]=\Psi\left[F\left(e_{i}\right)\right] \wedge \Psi\left[F\left(e_{j}\right)\right] \forall e_{i}, e_{j} \in A$.

Proposition 3.8. Homomorphic image of a modular $\ell$ - soft group is modular $\ell$ - soft group.

Proof. 
Let $\Psi:(F, A) \rightarrow(G, B)$ be $\ell$ - soft group onto homomorphism and suppose $(F, A)$ is modular $\ell$ - soft group.

Let $G\left(b_{1}\right), G\left(b_{2}\right), G\left(b_{3}\right) \in(G, B)$ with $G\left(b_{1}\right) \supseteq G\left(b_{2}\right) \supseteq G\left(b_{3}\right)$.

Since $\Psi$ is $\ell$ - soft group onto homomorphism, there exists $F\left(a_{1}\right)$, $F\left(a_{2}\right), F\left(a_{3}\right) \in(F, A)$ such that $\Psi\left(F\left(a_{1}\right)\right)=G\left(b_{1}\right), \Psi\left(F\left(a_{2}\right)\right)=$ $G\left(b_{2}\right), \Psi\left(F\left(a_{3}\right)\right)=G\left(b_{3}\right)$ where $F\left(a_{1}\right) \supseteq F\left(a_{2}\right)$.

Now $(F, A)$ is modular $\ell$ - soft group, $F\left(a_{1}\right), F\left(a_{2}\right), F\left(a_{3}\right) \in(F, A)$, we have

$F\left(a_{1}\right) \wedge\left[F\left(a_{2}\right) \vee F\left(a_{3}\right)\right]=\left[\left(F\left(a_{1}\right) \wedge F\left(a_{2}\right)\right] \vee\left[F\left(a_{1}\right) \wedge F\left(a_{3}\right)\right]\right.$

$=F\left(a_{2}\right) \vee\left[F\left(a_{1}\right) \wedge F\left(a_{3}\right)\right]$.

Now $G\left(b_{1}\right) \wedge\left[G\left(b_{2}\right) \vee G\left(b_{3}\right)\right]=\Psi\left(F\left(a_{1}\right)\right) \wedge\left[\Psi\left(F\left(a_{2}\right)\right) \vee \Psi\left(F\left(a_{3}\right)\right)\right]$

$=\Psi\left(F\left(a_{1}\right)\right) \wedge\left[\Psi\left(F\left(a_{2}\right) \vee\left(F\left(a_{3}\right)\right)\right]\right.$

$=\Psi\left[F\left(a_{1}\right) \wedge\left(\left(F\left(a_{2}\right) \vee\left(F\left(a_{3}\right)\right)\right]\right.\right.$

$=\Psi\left[\left(F\left(a_{2}\right) \vee\left(\left(F\left(a_{1}\right) \wedge\left(F\left(a_{3}\right)\right)\right]\right.\right.\right.$

$=\Psi\left(F\left(a_{2}\right)\right) \vee \Psi\left[\left(F\left(a_{1}\right) \wedge\left(F\left(a_{3}\right)\right]\right.\right.$

$=\Psi\left(\left(F\left(a_{2}\right)\right) \vee\left[\Psi\left(\left(F\left(a_{1}\right)\right) \wedge \Psi\left(\left(F\left(a_{3}\right)\right)\right]\right.\right.\right.$

$=G\left(b_{2}\right) \wedge\left[G\left(b_{1}\right) \vee G\left(b_{3}\right)\right]$.

Therefore $(G, B)$ is modular $\ell$ - soft group.

Definition 3.9. Let $(F, A)$ be a $\ell$ - soft group. A soft subset $(I, B)$ of $(F, A)$ is called a soft group $\ell$-ideal( $s g$ l-ideal), denoted by $(I, B) \unlhd_{\ell}(F, A)$, if the following are satisfied:

1. $B \subseteq A$, and for all $b_{1}, b_{2} \in B, b_{1} \vee b_{2}$ and $b_{1} \wedge b_{2}$ exist in $B$.

2. For all $I\left(b_{1}\right), I\left(b_{2}\right) \in(I, B), I\left(b_{1}\right) \vee I\left(b_{2}\right)$ and $I\left(b_{1}\right) \wedge I\left(b_{2}\right) e x$ ist in $(I, B)$.

3. $F(a) \in(F, A), I(b) \in(I, B)$ implies that $F(a) \wedge I(b)$ exists in $(I, B)$.

Definition 3.10. Let $(F, A)$ be a $\ell$ - soft group. A soft subset $(I, B)$ of $(F, A)$ is called a soft group dual $\ell$-ideal( sg dual $\ell$-ideal), denoted by $(I, B) \unrhd_{\ell}(F, A)$, if the following are satisfied:

1. $B \subseteq A$ and $\forall b_{1}, b_{2} \in B, b_{1} \vee b_{2}$ and $b_{1} \wedge b_{2}$ exist in $B$.

2. For all $I\left(b_{1}\right), I\left(b_{2}\right) \in(I, B), I\left(b_{1}\right) \vee I\left(b_{2}\right)$ and $I\left(b_{1}\right) \wedge I\left(b_{2}\right) e x$ ist in $(I, B)$.

3. $F(a) \in(F, A), I(b) \in(I, B)$ implies that $F(a) \vee I(b)$ exists in $(I, B)$.

Proposition 3.11. Let $(I, B) \tilde{\subseteq}(F, A)$. $(I, B)$ is a sg dual $\ell$ - ideal iff

1. $I\left(b_{1}\right), I\left(b_{2}\right) \in(I, B)$ implies $I\left(b_{1}\right) \wedge I\left(b_{2}\right) \in(I, B)$

2. $I\left(b_{1}\right) \in(I, B)$ and $I\left(b_{1}\right) \subseteq I\left(b_{2}\right)$ implies $I\left(b_{2}\right) \in(I, B)$

Proposition 3.12. An $\ell$ - soft group $(F, A)$ is modular $\ell$ - soft group iff $\mathscr{I}_{s g}(L)$, the soft group $\ell$-ideal of $(F, A)$ is modular $\ell$ - soft group. Proof.

Let $(F, A)$ be modular $\ell$ - soft group.

Let $(I, B),(J, D),(K, X) \in \mathscr{I}_{s g}(L)$.

To prove: $(I, B) \cap\left[(J, D) \cup_{R}(K, X)\right]=(J, D) \cup_{R}[(I, B) \cap(K, X)]$

Let $(J, D) \cup_{R}(K, X)=(H, E)$ where $E=D \cap X$ and let $(I, B) \cap$

$(H, E)=(M, Y)$, where $Y=B \cap E$

Let $M(y) \in(M, Y)=(I, B) \cap\left[(J, D) \cup_{R}(K, X)\right]$.

Then $M(y) \in(I, B)$ and $M(y) \in(H, E)$

$\Rightarrow M(y) \in(I, B)$ and $M(y) \in(J, D) \cup_{R}(K, X)$

$\Rightarrow M(y) \in(I, B)$ and $M(y) \in J(d) \vee K(x)$ for some $J(d) \in(J, D)$ and

$K(x) \in(K, X)$

since $J(d) \in(J, D) \tilde{\subseteq}(I, B), M(y) \vee J(d) \in(I, B)$.

Let $M(y) \vee J(d)=I(b)$, for some $I(b) \in(I, B)$

Now $M(y) \subseteq J(d) \vee K(x), M(y) \subseteq I(b)$.

$\Rightarrow M(y) \subseteq I(b) \wedge[J(d) \vee K(x)]$

$\Rightarrow M(y) \subseteq J(d) \vee[I(b) \wedge K(x)]$ as $I(b) \supseteq J(d)$ and $(F, A)$ is modular

$\ell$ - soft group.

Again $I(b) \wedge K(x) \subseteq I(b), I(b) \in(I, B)$

$\Rightarrow I(b) \wedge K(x) \in(I, B)$

$\Rightarrow I(b) \wedge K(x) \subseteq K(x), K(x) \in(K, X)$ [since Proposition:3.11]

$\Rightarrow I(b) \wedge K(x) \in(K, X)$.

Thus $I(b) \wedge K(x) \in(I, B) \cap(K, X)$ and $J(d) \in(J, D)$ implies $M(y) \in$

$(J, D) \cup_{R}[(I, B) \cap(K, X)]$ that is $(I, B) \cap\left[(J, D) \cup_{R}(K, D)\right] \subseteq(J, D) \cup_{R}[(I, B) \cap(K, X)]$ simillarly we prove $(J, D) \cup_{R}[(I, B) \cap(K, X)] \subseteq(I, B) \cap\left[(J, D) \cup_{R}\right.$ $(K, D)]$

Hence $\mathscr{I}_{\text {sg }}(L)$ is modular $\ell$ - soft group.

Coversely, let $\mathscr{I}_{s g}(L)$ is modular $\ell$ - soft group.

Define $\Psi:(F, A) \rightarrow \mathscr{I}_{\text {sg }}(L)$, such that $\Psi(F(a))=(F(a)]$, the principal sg $\ell$ - ideal generated by $F(a)$.

$\Psi\left(F\left(a_{1}\right)\right)=\Psi\left(F\left(a_{2}\right)\right)$

$\left.\Rightarrow\left(F\left(a_{1}\right)\right]=F\left(a_{2}\right)\right]$, then $\Psi$ is clearly 1-1 onto.

Also $\Psi\left(F\left(a_{1}\right) \wedge F\left(a_{2}\right)\right)=\left(F\left(a_{1}\right) \wedge F\left(a_{2}\right)\right]=\left(F\left(a_{1}\right)\right] \wedge\left(F\left(a_{2}\right)\right]=$ $\Psi\left(F\left(a_{1}\right)\right) \wedge \Psi\left(F\left(a_{2}\right)\right)$

$\Psi\left(F\left(a_{1}\right) \vee F\left(a_{2}\right)\right)=\left(F\left(a_{1}\right) \vee F\left(a_{2}\right)\right]=\left(F\left(a_{1}\right)\right] \vee\left(F\left(a_{2}\right)\right]=$ $\Psi\left(F\left(a_{1}\right)\right) \vee \Psi\left(F\left(a_{2}\right)\right)$

Hence $(F, A)$ can be imbedded into $\mathscr{I}_{\text {sg }}(L)$.

Therefore $(F, A)$ is modular $\ell$ - soft group.

Proposition 3.13. An $\ell$ - soft group $(F, A)$ is modular $\ell$ - soft group iff for $F\left(a_{1}\right), F\left(a_{2}\right), F\left(a_{3}\right) \in(F, A)$, the three relations $F\left(a_{1}\right) \supseteq F\left(a_{2}\right), \quad F\left(a_{1}\right) \wedge F\left(a_{3}\right)=F\left(a_{2}\right) \wedge F\left(a_{3}\right)$, $F\left(a_{1}\right) \vee F\left(a_{3}\right)=F\left(a_{2}\right) \vee F\left(a_{3}\right) \Rightarrow F\left(a_{1}\right)=F\left(a_{2}\right)$

Proof.

Let $(F, A)$ is modular $\ell$ - soft group and suppose $F\left(a_{1}\right), F\left(a_{2}\right), F\left(a_{3}\right) \in(F, A)$ are such that $F\left(a_{1}\right) \supseteq F\left(a_{2}\right)$, $F\left(a_{1}\right) \wedge F\left(a_{3}\right)=F\left(a_{2}\right) \wedge F\left(a_{3}\right)$ and $F\left(a_{1}\right) \vee F\left(a_{3}\right)=$ $F\left(a_{2}\right) \vee F\left(a_{3}\right)$.

Then $F\left(a_{1}\right)=F\left(a_{1}\right) \wedge\left[F\left(a_{1}\right) \vee F\left(a_{3}\right)\right]$ ( by absorption)

$=F\left(a_{1}\right) \wedge\left[F\left(a_{2}\right) \vee F\left(a_{3}\right)\right]$ ( by modularity)

$=F\left(a_{2}\right) \vee\left[F\left(a_{2}\right) \wedge F\left(a_{3}\right)\right]=F\left(a_{2}\right)$

Conversely, suppose the given conditions holds.

Let $F\left(a_{1}\right), F\left(a_{2}\right), F\left(a_{3}\right) \in(F, A), F\left(a_{1}\right) \supseteq F\left(a_{2}\right)$.

Now $\left\{F\left(a_{1}\right) \wedge\left[F\left(a_{2}\right) \vee F\left(a_{3}\right)\right\} \wedge F\left(a_{3}\right)=F\left(a_{1}\right) \wedge\left\{\left[F\left(a_{2}\right) \vee\right.\right.\right.$ $\left.\left.F\left(a_{2}\right) \vee F\left(a_{3}\right)\right] \wedge F\left(a_{3}\right)\right\}$

$=F\left(a_{1}\right) \wedge F\left(a_{3}\right)$ (by absorption)_-

Also $\left[F\left(a_{1}\right) \wedge F\left(a_{3}\right)\right]=\left[F\left(a_{1}\right) \wedge F\left(a_{3}\right)\right] \wedge F\left(a_{3}\right)$

$=\left\{F\left(a_{2}\right) \vee\left[F\left(a_{1}\right) \wedge F\left(a_{3}\right)\right]\right\} \wedge F\left(a_{3}\right)=F\left(a_{1}\right) \wedge F\left(a_{3}\right)$

that is, $\left\{F\left(a_{2}\right) \vee\left[F\left(a_{1}\right) \wedge F\left(a_{3}\right)\right]\right\} \wedge F\left(a_{3}\right)=F\left(a_{1}\right) \wedge F\left(a_{3}\right)$ - (ii)

Similarly, $\left\{F\left(a_{2}\right) \vee\left[F\left(a_{1}\right) \wedge F\left(a_{3}\right)\right]\right\} \vee F\left(a_{3}\right)=F\left(a_{2}\right) \vee\left\{\left[F\left(a_{1}\right) \wedge\right.\right.$

$\left.\left.F\left(a_{3}\right)\right] \vee F\left(a_{3}\right)\right\}=F\left(a_{2}\right) \vee F\left(a_{3}\right)-$ - (iii)

and $F\left(a_{2}\right) \vee F\left(a_{3}\right)=\left[F\left(a_{2}\right) \vee F\left(a_{3}\right)\right] \vee F\left(a_{3}\right)$

$=\left\{F\left(a_{1}\right) \wedge\left[F\left(a_{2}\right) \vee F\left(a_{3}\right)\right]\right\} \vee F\left(a_{3}\right)=F\left(a_{2}\right) \vee F\left(a_{3}\right)$

that is, $\left\{F\left(a_{1}\right) \wedge\left[F\left(a_{2}\right) \vee F\left(a_{3}\right)\right]\right\} \vee F\left(a_{3}\right)=F\left(a_{2}\right) \vee F\left(a_{3}\right)$

(iv)

From (i), (ii), (iii) and (iv), we have

$\left\{F\left(a_{1}\right) \wedge\left[F\left(a_{2}\right) \vee F\left(a_{3}\right)\right\} \wedge F\left(a_{3}\right)=\left\{F\left(a_{2}\right) \vee\left[F\left(a_{1}\right) \wedge F\left(a_{3}\right)\right]\right\} \wedge\right.$ $F\left(a_{3}\right)$

$\left\{F\left(a_{1}\right) \wedge\left[F\left(a_{2}\right) \vee F\left(a_{3}\right)\right\} \vee F\left(a_{3}\right)=\left\{F\left(a_{2}\right) \vee\left[F\left(a_{1}\right) \wedge F\left(a_{3}\right)\right]\right\} \vee\right.$

$F\left(a_{3}\right)$

Hence $F\left(a_{1}\right) \wedge\left[F\left(a_{2}\right) \vee F\left(a_{3}\right)\right]=F\left(a_{2}\right) \vee\left[F\left(a_{1}\right) \wedge F\left(a_{3}\right)\right]$

Proposition 3.14. $(F, A)$ is distributive $\ell$ - soft group iff $(F, A)$ satisfies soft median property. That is $\left[F\left(a_{1}\right) \vee F\left(a_{2}\right)\right] \wedge\left[F\left(a_{2}\right) \vee\right.$ $\left.F\left(a_{3}\right)\right] \wedge\left[F\left(a_{3}\right) \vee F\left(a_{1}\right)\right]=\left[F\left(a_{1}\right) \wedge F\left(a_{2}\right)\right] \vee\left[F\left(a_{2}\right) \wedge F\left(a_{3}\right)\right] \vee$ $\left[F\left(a_{3}\right) \wedge F\left(a_{1}\right)\right], \forall F\left(a_{1}\right), F\left(a_{2}\right), F\left(a_{3}\right) \in(F, A)$.

Proof.

Let $(F, A)$ is distributive $\ell$ - soft group.

$\left[F\left(a_{1}\right) \vee F\left(a_{2}\right)\right] \wedge\left[F\left(a_{2}\right) \vee F\left(a_{3}\right)\right] \wedge\left[F\left(a_{3}\right) \vee F\left(a_{1}\right)\right]$

$=\left\{F\left(a_{1}\right) \wedge\left\{\left[F\left(a_{2}\right) \vee F\left(a_{3}\right)\right] \wedge\left[F\left(a_{3}\right) \vee F\left(a_{1}\right)\right]\right\}\right\} \vee\left\{F\left(a_{2}\right) \wedge\right.$ $\left.\left\{\left[F\left(a_{2}\right) \vee F\left(a_{3}\right)\right] \wedge\left[F\left(a_{3}\right) \vee F\left(a_{1}\right)\right]\right\}\right\}$

$=\left\{\left[F\left(a_{1}\right) \wedge\left(F\left(a_{3}\right) \vee F\left(a_{1}\right)\right)\right] \wedge\left[F\left(a_{2}\right) \vee F\left(a_{3}\right)\right]\right\} \vee\left\{\left[F\left(a_{2}\right) \wedge\right.\right.$ $\left.\left.\left(F\left(a_{2}\right) \vee F\left(a_{3}\right)\right)\right] \wedge\left[F\left(a_{3}\right) \vee F\left(a_{1}\right)\right]\right\}$

$=\left\{\left[F\left(a_{1}\right) \wedge\left[F\left(a_{2}\right) \vee F\left(a_{3}\right)\right]\right\} \vee\left\{\left[F\left(a_{2}\right) \wedge\left[F\left(a_{3}\right) \vee F\left(a_{1}\right)\right]\right\}\right.\right.$

$=\left[F\left(a_{1}\right) \wedge F\left(a_{2}\right)\right] \vee\left[F\left(a_{1}\right) \wedge F\left(a_{3}\right)\right] \vee\left[F\left(a_{2}\right) \wedge F\left(a_{3}\right)\right] \vee\left[F\left(a_{2}\right) \wedge\right.$

$\left.F\left(a_{1}\right)\right]$

$=\left[F\left(a_{1}\right) \wedge F\left(a_{2}\right)\right] \vee\left[F\left(a_{2}\right) \wedge F\left(a_{3}\right)\right] \vee\left[F\left(a_{3}\right) \wedge F\left(a_{1}\right)\right]$

Conversely, first to prove $(F, A)$ is modular $\ell$ - soft group,

Let $F\left(a_{1}\right), F\left(a_{2}\right), F\left(a_{3}\right) \in(F, A)$, with $F\left(a_{1}\right) \supseteq F\left(a_{2}\right)$.

Then

$F\left(a_{1}\right) \wedge\left[F\left(a_{2}\right) \vee F\left(a_{3}\right)\right]=\left[F\left(a_{1}\right) \wedge\left(F\left(a_{1}\right) \vee F\left(a_{3}\right)\right)\right] \wedge\left(F\left(a_{2}\right) \vee\right.$ 


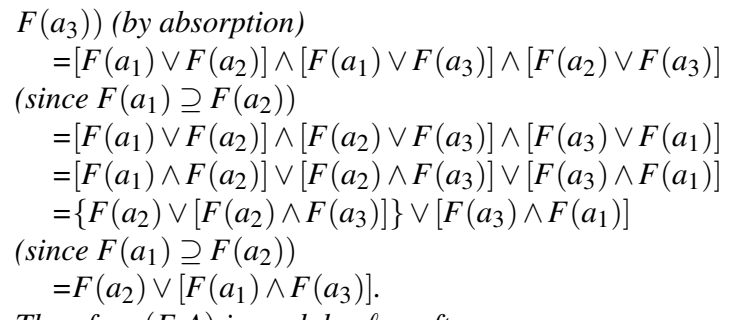

Therefore $(F, A)$ is modular $\ell$ - soft group.

Now for any $F\left(a_{1}\right), F\left(a_{2}\right), F\left(a_{3}\right) \in(F, A)$,

$F\left(a_{1}\right) \wedge\left[F\left(a_{2}\right) \vee F\left(a_{3}\right)\right]=\left[F\left(a_{1}\right) \wedge\left(F\left(a_{1}\right) \vee F\left(a_{3}\right)\right)\right] \wedge\left[F\left(a_{2}\right) \vee\right.$ $\left.F\left(a_{3}\right)\right]$

$=\left[F\left(a_{1}\right) \wedge\left(F\left(a_{1}\right) \vee F\left(a_{2}\right)\right) \wedge\left(F\left(a_{1}\right) \vee F\left(a_{3}\right)\right)\right] \wedge\left[F\left(a_{2}\right) \vee F\left(a_{3}\right)\right]$ $=F\left(a_{1}\right) \wedge\left[\left(F\left(a_{1}\right) \vee F\left(a_{2}\right)\right) \wedge\left(F\left(a_{2}\right) \vee F\left(a_{3}\right)\right) \wedge\left(F\left(a_{3}\right) \vee F\left(a_{1}\right)\right]\right.$ $=F\left(a_{1}\right) \wedge\left[\left(F\left(a_{1}\right) \wedge F\left(a_{2}\right)\right) \vee\left(F\left(a_{2}\right) \wedge F\left(a_{3}\right)\right) \vee\left(F\left(a_{3}\right) \wedge F\left(a_{1}\right)\right]\right.$ $=F\left(a_{1}\right) \wedge\left[\left(F\left(a_{2}\right) \wedge F\left(a_{3}\right)\right) \vee\left(F\left(a_{1}\right) \wedge F\left(a_{2}\right)\right) \vee\left(F\left(a_{3}\right) \wedge F\left(a_{1}\right)\right]\right.$ Now by using modular $\ell$ - soft group and as $F\left(a_{1}\right) \supseteq F\left(a_{1}\right) \wedge F\left(a_{2}\right)$, $F\left(a_{1}\right) \supseteq F\left(a_{3}\right) \wedge F\left(a_{1}\right)$ gives $F\left(a_{1}\right) \supseteq\left[F\left(a_{1}\right) \wedge F\left(a_{2}\right)\right] \vee\left[F\left(a_{3}\right) \wedge\right.$ $\left.F\left(a_{1}\right)\right]$.

Then $F\left(a_{1}\right) \wedge\left[F\left(a_{2}\right) \vee F\left(a_{3}\right)\right]=\left[\left(F\left(a_{1}\right) \wedge F\left(a_{2}\right)\right) \vee\left(F\left(a_{3}\right) \wedge\right.\right.$ $\left.\left.F\left(a_{1}\right)\right)\right] \vee$

$\left[\left(F\left(a_{2}\right) \wedge F\left(a_{3}\right)\right) \wedge F\left(a_{1}\right)\right]$

$=\left(F\left(a_{1}\right) \wedge F\left(a_{2}\right)\right) \vee\left\{\left(F\left(a_{3}\right) \wedge F\left(a_{1}\right)\right) \vee\left[\left(F\left(a_{3}\right) \wedge F\left(a_{1}\right)\right) \wedge F\left(a_{2}\right)\right]\right\}$

$=\left[F\left(a_{1}\right) \wedge F\left(a_{2}\right)\right] \vee\left[\left(F\left(a_{3}\right) \wedge F\left(a_{1}\right)\right]\right.$

Therefore $(F, A)$ is distributive $\ell$ - soft group.

Proposition 3.15. A sub $\ell$ - soft group of a distributive $\ell$ - soft group is distributive $\ell$ - soft group.

Proposition 3.16. Homomorphic image of a distributive $\ell$ - soft group is distributive $\ell$ - soft group.

Proposition 3.17. $\mathscr{I}_{s g}(L)$, the soft group $\ell$-ideal of $(F, A)$ is distributive $\ell$ - soft group iff $(F, A)$ is distributive $\ell$ - soft group.

Proposition 3.18. An $\ell$ - soft group $(F, A)$ is distributive $\ell$ - soft group iff for $F\left(a_{1}\right), F\left(a_{2}\right), F\left(a_{3}\right) \in(F, A), F\left(a_{1}\right) \wedge F\left(a_{3}\right)=F\left(a_{2}\right) \wedge$ $F\left(a_{3}\right)$ and $F\left(a_{1}\right) \vee F\left(a_{3}\right)=F\left(a_{2}\right) \vee F\left(a_{3}\right) \Rightarrow F\left(a_{1}\right)=F\left(a_{2}\right)$.

Proof.

Let $(F, A)$ is distributive $\ell$ - soft group and let $F\left(a_{1}\right) \wedge F\left(a_{3}\right)=$ $F\left(a_{2}\right) \wedge F\left(a_{3}\right)$ and $F\left(a_{1}\right) \vee F\left(a_{3}\right)=F\left(a_{2}\right) \vee F\left(a_{3}\right)$.

Then $F\left(a_{1}\right)=F\left(a_{1}\right) \wedge\left[F\left(a_{1}\right) \vee F\left(a_{3}\right)\right]$ ( by absorption)

$=F\left(a_{1}\right) \wedge\left[F\left(a_{2}\right) \vee F\left(a_{3}\right)\right]$

$=\left[F\left(a_{1}\right) \wedge F\left(a_{2}\right)\right] \vee\left[F\left(a_{1}\right) \wedge F\left(a_{3}\right)\right]$

$=\left[F\left(a_{1}\right) \wedge F\left(a_{2}\right)\right] \vee\left[F\left(a_{2}\right) \wedge F\left(a_{3}\right)\right]$

$=F\left(a_{2}\right) \wedge\left[F\left(a_{1}\right) \vee F\left(a_{3}\right)\right]$

$=F\left(a_{2}\right) \wedge\left[F\left(a_{2}\right) \vee F\left(a_{3}\right)\right]=F\left(a_{2}\right)$

Conversely, $F\left(a_{1}\right) \wedge F\left(a_{3}\right)=F\left(a_{2}\right) \wedge F\left(a_{3}\right)$ and $F\left(a_{1}\right) \vee F\left(a_{3}\right)=$ $F\left(a_{2}\right) \vee F\left(a_{3}\right) \Rightarrow F\left(a_{1}\right)=F\left(a_{2}\right), \forall F\left(a_{1}\right), F\left(a_{2}\right), F\left(a_{3}\right) \in(F, A)$.

By the proposition 3.13, $(F, A)$ is modular $\ell$ - soft group with $F\left(a_{1}\right) \supseteq$ $F\left(a_{2}\right)$.

Hence $(F, A)$ is distributive $\ell$ - soft group.

Theorem 3.19. Let $(F, A)$ be a soft $\ell$-modular and $F\left(a_{1}\right), F\left(a_{2}\right) \in$ $(F, A)$ Then there exist non-empty inerval in $(F, A)$ such that $\left[F\left(a_{1}\right) \wedge\right.$ $\left.F\left(a_{2}\right), F\left(a_{1}\right)\right] \cong\left[F\left(a_{2}\right), F\left(a_{1}\right) \vee F\left(a_{2}\right)\right]$.

Proof:

Any non-empty interval in an $\ell$ - soft group is a sub $\ell$ - soft group. Define a map $\chi:\left[F\left(a_{1}\right) \wedge F\left(a_{2}\right), F\left(a_{1}\right)\right] \rightarrow\left[F\left(a_{2}\right), F\left(a_{1}\right) \vee\right.$ $\left.F\left(a_{2}\right)\right]$ such that $\chi\left(F\left(a_{3}\right)\right)=F\left(a_{3}\right) \vee F\left(a_{2}\right), F\left(a_{3}\right) \in\left[F\left(a_{1}\right) \wedge\right.$ $\left.F\left(a_{2}\right), F\left(a_{1}\right)\right]$.

(i) $\chi$ is well-defined:

$F\left(a_{3}\right) \in\left[F\left(a_{1}\right) \wedge F\left(a_{2}\right), F\left(a_{1}\right)\right] \Rightarrow F\left(a_{1}\right) \wedge F\left(a_{2}\right) \subseteq F\left(a_{3}\right) \subseteq F\left(a_{1}\right)$ $\Rightarrow\left[F\left(a_{1}\right) \wedge F\left(a_{2}\right)\right] \vee F\left(a_{2}\right) \subseteq F\left(a_{3}\right) \vee F\left(a_{2}\right) \subseteq F\left(a_{1}\right) \vee F\left(a_{2}\right)$

$\Rightarrow F\left(a_{2}\right) \subseteq F\left(a_{3}\right) \vee F\left(a_{2}\right) \subseteq F\left(a_{1}\right) \vee F\left(a_{2}\right)$

$\Rightarrow F\left(a_{3}\right) \vee F\left(a_{2}\right) \in\left[F\left(a_{2}\right), F\left(a_{1}\right) \vee F\left(a_{2}\right)\right]$
Also $F\left(a_{3}\right)=F\left(a_{4}\right) \Rightarrow F\left(a_{3}\right) \vee F\left(a_{2}\right)=F\left(a_{4}\right) \vee F\left(a_{2}\right) \Rightarrow$ $\chi\left[F\left(a_{3}\right)\right]=\chi\left[F\left(a_{4}\right)\right]$

(ii) $\chi$ is 1-1:

Let $F\left(a_{3}\right), F\left(a_{4}\right) \in\left[F\left(a_{1}\right) \wedge F\left(a_{2}\right), F\left(a_{1}\right)\right], \chi\left[F\left(a_{3}\right)\right]=\chi\left[F\left(a_{4}\right)\right]$

Then $F\left(a_{3}\right) \vee F\left(a_{2}\right)=F\left(a_{4}\right) \vee F\left(a_{2}\right)$

$\Rightarrow F\left(a_{1}\right) \wedge\left[F\left(a_{3}\right) \vee F\left(a_{2}\right)\right]=F\left(a_{1}\right) \wedge\left[F\left(a_{4}\right) \vee F\left(a_{2}\right)\right]$

$\Rightarrow F\left(a_{3}\right) \vee\left[F\left(a_{1}\right) \wedge F\left(a_{2}\right)\right]=F\left(a_{4}\right) \vee\left[F\left(a_{1}\right) \wedge F\left(a_{2}\right)\right]$

$\Rightarrow F\left(a_{3}\right)=F\left(a_{4}\right)$ because $F\left(a_{1}\right) \wedge F\left(a_{2}\right) \subseteq F\left(a_{3}\right), F\left(a_{4}\right)$

(iii) $\chi$ is onto:

Let $F\left(a_{5}\right) \in\left[F\left(a_{2}\right), F\left(a_{1}\right) \vee F\left(a_{2}\right)\right]$ be any arbitrary element.

Now $F\left(a_{5}\right) \in\left[F\left(a_{2}\right), F\left(a_{1}\right) \vee F\left(a_{2}\right)\right] \Rightarrow F\left(a_{2}\right) \subseteq F\left(a_{5}\right) \subseteq F\left(a_{1}\right) \vee$ $F\left(a_{2}\right) \Rightarrow F\left(a_{1}\right) \wedge F\left(a_{2}\right) \subseteq F\left(a_{1}\right) \wedge F\left(a_{5}\right) \subseteq F\left(a_{1}\right) \wedge\left[F\left(a_{1}\right) \vee\right.$ $\left.F\left(a_{2}\right)\right] \Rightarrow F\left(a_{1}\right) \wedge F\left(a_{5}\right) \in\left[F\left(a_{1}\right) \wedge F\left(a_{2}\right), F\left(a_{1}\right)\right]$

Also $\chi\left[F\left(a_{1}\right) \wedge F\left(a_{5}\right)\right]=\left[F\left(a_{1}\right) \wedge F\left(a_{5}\right)\right] \vee F\left(a_{2}\right)$

Now $\left.F\left(a_{5}\right) \subseteq F\left(a_{1}\right)\right] \vee F\left(a_{2}\right) \Rightarrow F\left(a_{5}\right) \wedge\left[F\left(a_{1}\right) \vee F\left(a_{2}\right)\right]=F\left(a_{5}\right)$

$\Rightarrow F\left(a_{5}\right)=F\left(a_{5}\right) \wedge\left[F\left(a_{2}\right) \vee F\left(a_{1}\right)\right]=F\left(a_{2}\right) \vee\left[F\left(a_{5}\right) \wedge F\left(a_{1}\right)\right]$

Hence $\chi$ is onto.

(iv) $\chi$ is an $\ell$ - soft group homomorphism:

$\chi\left[F\left(a_{1}\right) \vee F\left(a_{2}\right)\right]=F\left(a_{3}\right) \vee\left[F\left(a_{1}\right) \vee F\left(a_{2}\right)\right]=\left[F\left(a_{3}\right) \vee F\left(a_{1}\right)\right] \vee$

$\left[F\left(a_{3}\right) \vee F\left(a_{2}\right)\right]=\chi\left[F\left(a_{1}\right)\right] \vee \chi\left[F\left(a_{2}\right)\right]$ and

$\chi\left[F\left(a_{1}\right) \wedge F\left(a_{2}\right)\right]=F\left(a_{3}\right) \vee\left[F\left(a_{1}\right) \wedge F\left(a_{2}\right)\right]=\left[F\left(a_{3}\right) \vee F\left(a_{1}\right)\right] \wedge$

$\left[F\left(a_{3}\right) \vee F\left(a_{2}\right)\right]=\chi\left[F\left(a_{1}\right)\right] \vee \chi\left[F\left(a_{2}\right)\right]$

Hence $\left[F\left(a_{1}\right) \wedge F\left(a_{2}\right), F\left(a_{1}\right)\right] \cong\left[F\left(a_{2}\right), F\left(a_{1}\right) \vee F\left(a_{2}\right)\right]$

\section{Conclusion}

In this work, we have investigated the modular $\ell$ - soft group and distributive $\ell$ - soft group of $\ell$ - soft group and shown several related properties. From this, we could extend our work by applying $\ell$ soft group in different types of algebras such as heyting algebra, p-algebra, etc.

\section{Acknowledgement}

The authors are thankful to the respected referee for valuable comments in improving this work.

\section{References}

[1] H.Aktas and N.Cagman, 2007, Soft sets and soft groups, Inform.Sciences.177, pp.2726-2735.

[2] V.S.Anusuya Ilamathi and J.Vimala, 2018, Multi-criteria Decision Making on Lattice Ordered Multisets In: Thampi S., Mitra S., Mukhopadhyay J., Li KC., James A., Berretti S. (eds) Intelligent Systems Technologies and Applications. ISTA 2017. Advances in Intelligent Systems and Computing, vol 683. Springer, Cham. pp.401416

[3] J.Arockia Reeta and J.Vimala, 2016, A study on distributive and modular lattice ordered fuzzy soft group and its duality, Appl. Math. J. Chinese Univ., volume 31, 4, 491-502.

[4] M.I.Ali, Tahir Mahmood.Muti Ur Rehman, M.Fahim Aslam, 2015, On Lattice Ordered Soft Sets, Applied Soft Computing.36, pp.499-505.

[5] P.Bharathi and J.Vimala, 2016, The Role of Fuzzy l-ideals in a Commutative l-group, Global Journal of Pure and Applied Mathematics, volume 12, Number 3, pp. 2067 - 2074.

[6] P.Bharathi, J.Vimala, L.Vijayalakshmi and J.Arockia Reeta, 2017, Some Contributions of Congruence Relations on Lattices of Fuzzy l-ideal Appl. Math. inform. sci. II, No.2, pp. 1-5.

[7] W.H. Cornish, 1977, Characterization distributive and modular semilattices, Math. Japonica, 22 pp. 159-174.

[8] Faruk Karaaslan, Naim Cagman and Serdar Enginoglu, 2012, Soft Lattices, Journal of New Results in Science, No. 1, pp.5-17

[9] Garett Birkhoff, Lattice theory, American Mathematical Society Colloqium Publications, volume XXV.

[10] G. Gratzer, 1971, Lattice Theory: First Concepts and Distributive Lattices, Freeman and Company.

[11] Grzegorz Dymek and Andrzej Walendziak, 2015, Soft Set Theory Applied To General Algebras, Demonstratio Mathematica, Vol. XLVIII, No. 1 .

[12] P.K.Maji,R.Biswas,A.R.Roy, 2003, soft set theory, Comput.Math.Appl., 45, pp.555-562.

[13] D.A.Molodtsov, 1999, Soft set theory - First result, Comput. Math.Appl.,37, pp.19-31. 
[14] E.K.R.Nagarajan and P.Geetha, 2013, Characterization Theorems for Modular and Distributive Soft Lattices, Intnl.Jorl.of Innovative Research in science Eng. and Tech., 2, issue 11, pp. 2319-8753

[15] Natarajan.R and Vimala.J, 2007, Distributive l-ideal in a commutative l-group, Acta Ciencia Indica, Vol.XXXIII M.No.2.517, MR 2392259.

[16] Onyeozili I.A.,Gwary T.M, 2014, A Study on the Fundamentals of Soft Set Theory, International Journal of Scientific and Technology Research, Volume 3, issue 4, pp.132-143.

[17] L.Vijayalakshmi and J.Vimala, 2017, On Lattice Ordered Soft Groups, International Journal of Pure and Applied Mathematics vol.112,issue 1 Article No.AP2016-31-108, pp. 47-55

[18] J.Vimala, 2014, Fuzzy Lattice Ordered Group, International Journal of Scientific and Engineering Research, Volume 5, Issue 9, pp.58-60

[19] J.Vimala and J.Arockia Reeta, 2016, A study on lattice ordered fuzzy soft group, International Journal of Applied Mathematical Sciences, volume 9, No.1, pp.1-10.

[20] J.Vimala, J.Arockia Reeta and V.S.Anusuya Ilamathi, A study on Fuzzy Soft Cardinality in Lattice Ordered Fuzzy Soft Group and its application in Decision Making Problems, Journal of Intelligent and Fuzzy Systems-IOS Press (Accepted).

[21] Xia Yin, Zuhua Liao, 2013, Study on Soft Groups, Journal of Comp. vol.8.

[22] Yingehao Shao,Keyun Qin, 2011, The Lattice structure of the soft groups, Procedia Engineering 15, pp.3621-3625. 